\title{
1 Two dominant brain states reflect optimal and suboptimal attention
}

Ayumu Yamashita ${ }^{1,2^{*}}$, David Rothlein ${ }^{1,2}$, Aaron Kucyi ${ }^{3}$, Eve M. Valera ${ }^{4}$, and Michael Esterman ${ }^{1,2,5}$

${ }^{1}$ Department of Psychiatry, Boston University School of Medicine, Massachusetts, United States.

${ }^{2}$ Boston Attention and Learning Laboratory, VA Boston Healthcare System, Massachusetts, United States.

${ }^{3}$ Department of Psychology, Northeastern University, Massachusetts, United States.

${ }^{4}$ Department of Psychiatry, Harvard Medical School, Massachusetts, United States.

${ }^{5}$ National Center for PTSD, VA Boston Healthcare System, Massachusetts, United States.

* Corresponding author

\section{Abstract}

Attention is not constant but fluctuates from moment to moment. Previous studies dichotomized these fluctuations into optimal and suboptimal states based on behavioral performance and investigated the difference in brain activity between these states. Although these studies implicitly assume there are two states, this assumption is not guaranteed. Here, we reversed the logic of these previous studies and identified unique states of brain activity during a sustained attention task. We demonstrate a systematic relationship between dynamic brain activity patterns (brain states) and behavioral underpinnings of sustained attention by explaining behavior from two dominantly observed brain states. In four independent datasets, a brain state characterized by default mode network activity was behaviorally optimal and a brain state characterized by dorsal attention network activity was suboptimal. Thus, our study provides compelling evidence for behaviorally optimal and suboptimal attentional states from the sole viewpoint of brain activity. We further demonstrated how these brain states were impacted by motivation, mind wandering, and attention-deficit hyperactivity disorder. Within-subject level modulators (motivation and mind wandering) impacted the optimality of behavior in the suboptimal brain state. In contrast, between-subject level differences (ADHD vs healthy controls) impacted the optimal brain state character, namely its frequency. 


\section{$1 \quad$ Introduction}

2 Attention is not constant but fluctuates from moment to moment ${ }^{1-4}$. The elucidation of the

3 brain mechanisms required to sustain attention is theoretically important and translationally

4 relevant $^{5-11}$. A wealth of previous studies dichotomized these fluctuations into optimal (stable

5 reaction times, fast reaction times, or fewer attentional lapses/errors) and suboptimal

6 (variable reaction times, slow reaction times, or more attentional lapses/errors) states based

7 on behavioral performance and investigated the differences in brain activity between these

8 behaviorally inferred attentional states ${ }^{6,7,12-21}$. Almost all studies have reported contrasting

9 brain-behavior relationships with task negative networks such as default mode network

10 (DMN) and attention related networks such as dorsal attention network (DAN) and salience

11 network (SN). In a growing number of studies, optimal performance states were associated

12 with greater DMN activity, while suboptimal performance states were associated with greater

13 DAN and SN activity ${ }^{7,18,21,22}$. Although the directionality of the relationship between brain

14 activity and sustained attention is controversial in previous studies, such approaches, which

15 infer mental states based on behavior, are limited by the low dimensionality of behavioral

16 variables-resulting in blunt methods like dichotomization to identify two separate states

17 when in reality, brain dynamics may be more complicated. Furthermore, such approaches

18 rely on frequent responses from participants, and this serves as a strong constraint on the

19 types of tasks that can be used to identify attentional states. To address these stark limitations

20 in the literature, we consider whether attentional states can be defined and observed on the

21 basis of brain activity alone.

Previous studies have also shown relationships between sustained attention ability

23 and the functional connectivity between functionally different brain systems $s^{10,11,18,23}$.

24 Functional connectivity is defined as temporal correlation between blood oxygen level

25 dependent (BOLD) time courses from functional magnetic resonance imaging (fMRI). For

26 example, participants who have stronger anticorrelation of functional connectivity between 
$1 \mathrm{DMN}$ and DAN/SN tend to perform better (more stable and faster reaction times) ${ }^{19,24}$.

2 Individual sustained attention ability also could be predicted by functional connectivity

3 patterns across entire parcellations of the brain (connectome) $)^{10,11,25}$. Furthermore, fMRI

4 connectivity neurofeedback training or stimulants such as Methylphenidate have been shown

5 to induce changes in both functional connectivity and sustained attention performance ${ }^{26,27}$.

6 These findings implicate the connectome of functionally different brain systems in supporting

7 sustained attention ability. Nonetheless, whether and how the connectome relates to sustained

8 attention performance through the intermediary of their dynamic brain activity patterns

9 remains unclear ${ }^{28}$.

10 Here we used a novel energy landscape analysis ${ }^{29-33}$ to identify dynamic brain

11 activity states in a manner that was agnostic to behavior during a sustained attention task.

12 Energy landscape analysis is a data-driven method for estimating stable brain states under the

13 constraints of the connectome between functionally different brain systems. We examined the

14 observed number of states, and whether performance during distinct states corresponded to

15 behaviorally defined optimal and/or suboptimal state(s) ${ }^{7,18,21}$. We provided additional support

16 for these results using an independent validation dataset for replication purposes. To extend

17 this result, we based an additional set of experiments on studies that have shown sustained

18 attention is improved by motivation ${ }^{34,35}$, worsened by mind wandering ${ }^{22}$, and impaired in

19 neuropsychiatric disorders of attention such as attention-deficit hyperactivity disorder

$20(\mathrm{ADHD})^{6,8-11}$. It remains unknown whether these positive (motivation) and negative (mind

21 wandering, neuropsychiatric disorder) modulators directly impact the composition of the

22 optimal and suboptimal brain states or rather impact the optimality of behavior or likelihood

23 of reaching a given brain state. Using three additional datasets, we investigated how

24 motivation, mind wandering, and ADHD impact the character of these brain states (activity

25 patterns and frequency of these states) and the relationship to performance during these states.

26 Overall, we demonstrate and validate a novel approach for defining attentional states across 
1 four independent datasets (see Methods). Further, we provide the first evidence for the

2 existence of and correspondence between neurally and behaviorally defined optimal and

3 suboptimal attentional states. Finally, we demonstrate various influences of positive and

4 negative factors on the character and optimality of these states.

$6 \quad$ Results

7 Local energy minimum brain states during gradual onset continuous performance task.

8 To investigate the connectome between functionally different brain systems, we first defined

914 region of interests (ROIs) which represent functionally different brain systems (Fig. 1a,b)

10 by applying dictionary learning ${ }^{36-38}$ to resting state fMRI with 16 participants (6 males, ages

$11 \quad 18-34$ years, mean age $=24.1$ years). Dictionary learning can extract brain spatial maps that

12 are naturally sparse and usually cleaner than independent component analysis. We then

13 prepared a time series of average BOLD time courses of 14 ROIs (functionally different brain

14 systems) while 16 participants performed the gradual onset continuous performance task

15 (gradCPT; Dataset 1). GradCPT is a well-validated test of sustained attention, previously

16 used to define attentional states defined by reaction time variability fluctuations over time ${ }^{7,21}$.

17 We then binarized the 14 ROIs' activity at each time point (by replacing all values above a

18 mean activation with active and others with inactive within each ROI), fitted a pairwise

19 maximum entropy model (MEM) $)^{31,39}$ to them, and derived functional connectivity matrices

20 (connectome) between ROIs and average activation of each ROI. Next, based on the

21 connectome between functionally different brain systems and average activation of each ROI,

22 we calculated energy values of all the possible brain activity patterns ( $2^{14}$ patterns) (see

23 Pairwise maximum entropy model section in the Methods). We then examined hierarchal

24 relationships between the $2^{14}$ energy values and systematically searched for dominant brain

25 activity patterns that showed locally minimum energy values that were more likely to be

26 observed than similar activity patterns ${ }^{29,30,32,33}$ (Fig. 1c). Please note that this energy value 
1 does not indicate any biological energy. It is rather a statistical index that indicates the

2 occurrence probability of each brain activity pattern. For instance, activity patterns with

3 lower energy values tend to occur more frequently. If dynamic brain activity during gradCPT

4 can be described as transitions between behaviorally optimal and suboptimal attention states ${ }^{21}$

5 (Fig. 1d), such states may correspond to stable brain states which have local energy

6 minimums.

As a result, we found that 13 stable brain states frequently occurred during gradCPT.

8 Figure 2a showed the brain activity patterns in the 14 ROIs for the 13 stable brain states.

9 Figure $2 \mathrm{~b}$ showed the percentage of dwell time during gradCPT. We found that both State 1

12 was characterized by DAN and SN activity.

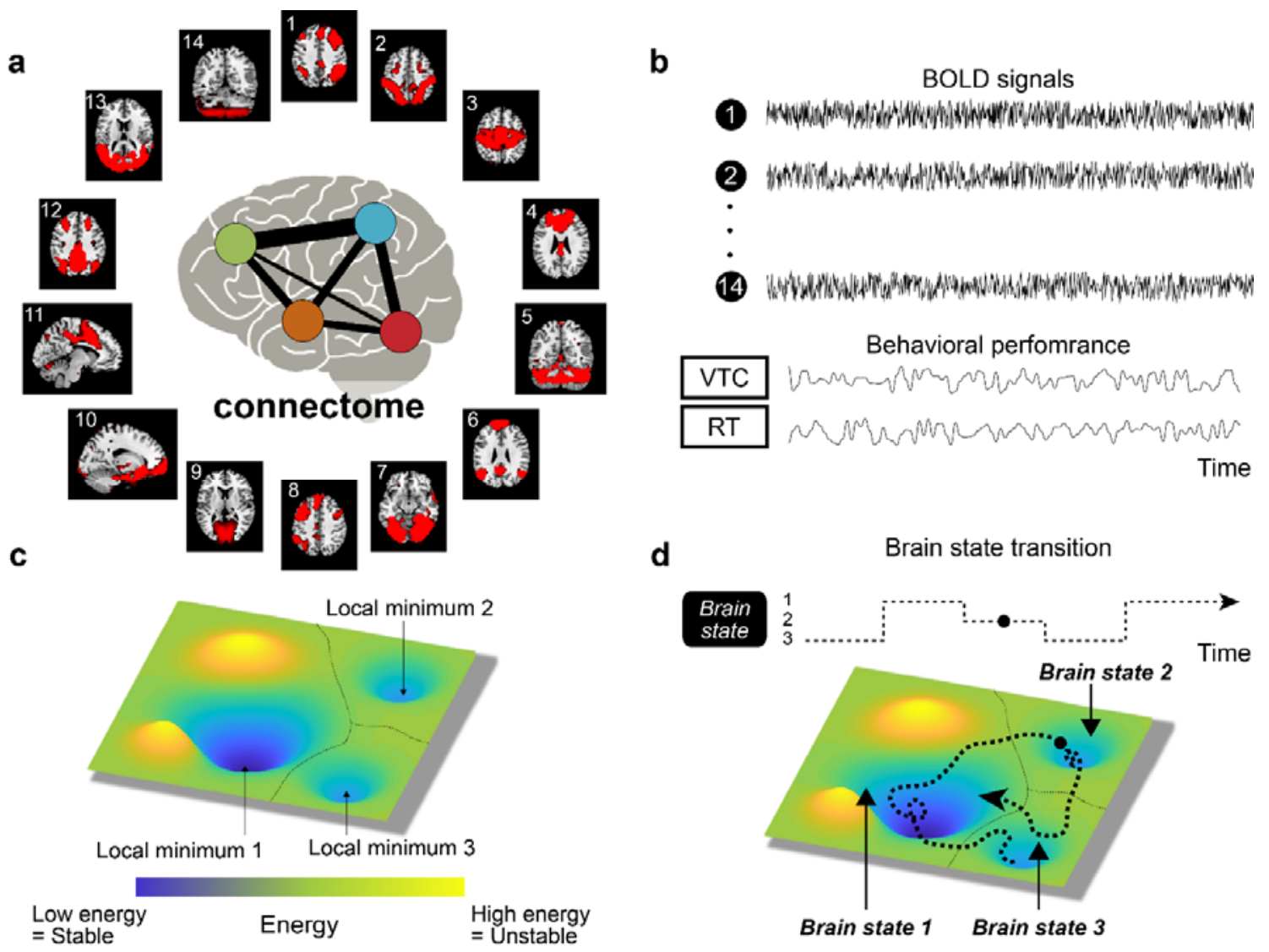

Figure 1. Procedures of energy landscape analysis. (a) Region of interests (ROIs) from dictionary learning using resting-state fMRI. These ROIs indicate functionally different brain regions. Connectome indicates functional connectivity pattern between 
ROIs. (b) BOLD signals extracted from 14 ROIs and behavioral performances in gradual onset continuous performance task. (c) Energy landscape and local minimums. (d) Brain state transition in energy landscape. BOLD: Blood oxygen level dependent; VTC: Variance time course; RT: Reaction time.

We characterized these dominant brain states by focusing on DMN, DAN and SN (ROI 6, 2, and 11 , respectively), based on the previous studies linking these functionally different brain systems to fluctuations in sustained attention ${ }^{7,21}$. We then summarized individual local minimum brain states into two major brain state categories (DMN-state and DAN-state) and others. The DMN-state was defined as DMN-active, DAN-inactive, and SN-inactive. The DAN-state was defined as DMN-inactive along with either or both DAN-active and $\mathrm{SN}$-active. If local minimum brain state did not fit into the above criteria, we defined such brain state as "other" ( e.g. both DMN and DAN or SN were active or DMN, DAN and SN were all inactive ). Using these rules, DMN-state and DAN-state covered $48 \%$ and $51 \%$ of total time, respectively (Fig.2b right). We further investigated whether these brain states were specific to the gradCPT. To this end, we investigated local minimum brain states during resting state fMRI. These two brain states existed even in resting state, but these were less dominant, covering $40 \%$ and $39 \%$ respectively (Figs. 2cd). Furthermore, we confirmed these two brain states were robust to choice of ROIs (Supplementary Figures 2 and 3) ) $^{40,41}$. For example, when we used the Schaefer et al. ${ }^{40} 200$ ROIs categorized by networks (7), the only states found were a DMN-state and a DAN+SN-state even without summarizing (Supplementary Figures 2). These results indicate that fluctuation of the brain activity during gradCPT can be described as dynamic transitions between two dominant brain states represented by DMN, DAN and SN activity patterns. 
a Stable brain activity pattern during gradCPT

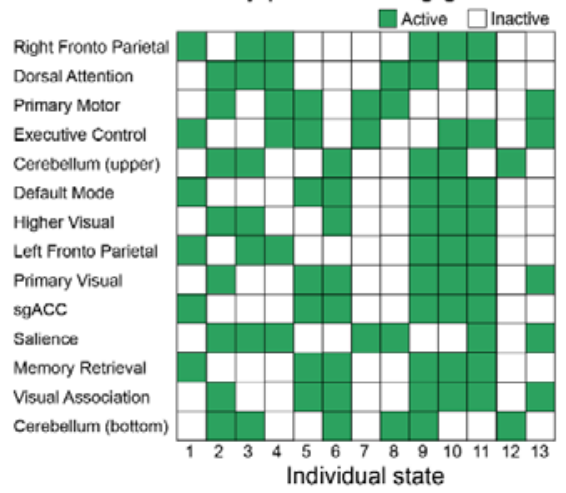

C Stable brain activity pattern during rest

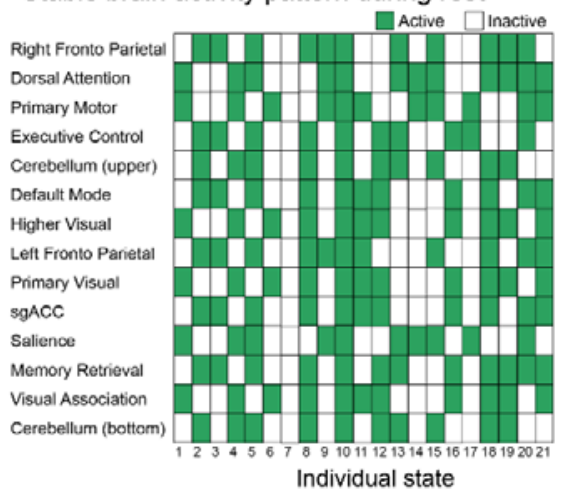

b

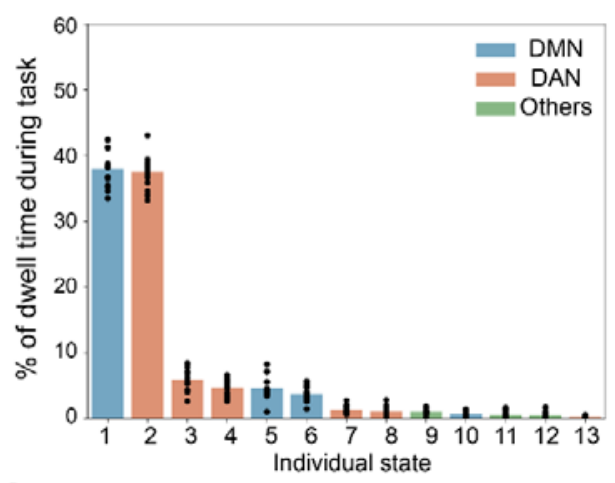

d

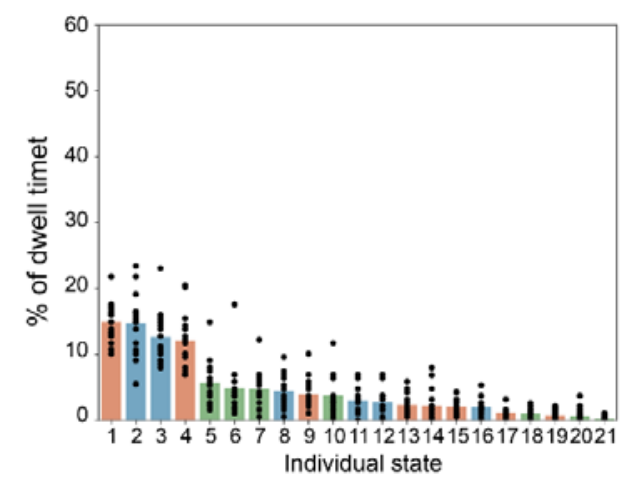

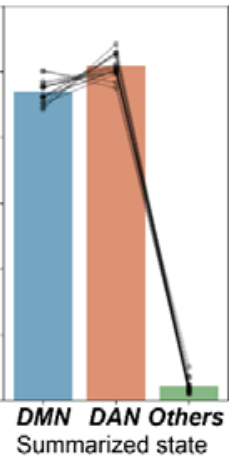

Summarized state

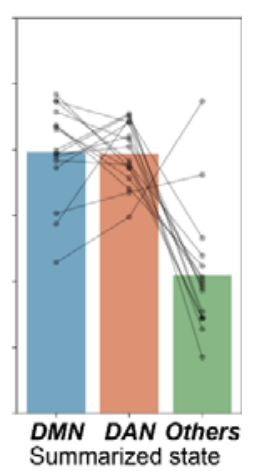

Figure 2. Energetically stable brain states and dwell time during task and rest. (a) Stable brain states during gradual onset continuous performance task (gradCPT). Individual state is represented by an activity pattern in which each brain region is active (green) or inactive (white) state. (b) Left graph shows percentage of dwell time during gradCPT in each individual state. Blue bars show the state defined as DMN-state, red bars show the state defined as DAN-state, green bar show the state defined as Others. Each scatter shows each participant. Right graph shows summation for each summarized state. (c) Stable brain states during resting state. (d) Percentage of dwell time during resting state. sgACC: subgenual anterior cingulate cortex; DMN: default mode network; DAN: dorsal attention network.

We then examined behavioral differences in performances between the DMN-state and DAN-state. We focused on variables commonly used to assess the optimality of sustained attention, including mean reaction time, mean variance time course (VTC, a measure of reaction time variability), and accuracy (d prime) as measures of performance (Fig. 1b). We shifted the time labels of the brain states backwards by 5 seconds to account for the hemodynamic response lag. We found that mean VTC and d prime were significantly better 
1 (lower variability and higher accuracy) during timepoints corresponding to the DMN-state

2 than those in DAN-state (Wilcoxon signed-rank test. Variance time course: $W_{15}=21, P<$

3 0.016; Reaction time: $W_{15}=39, P>0.13$; d prime: $W_{15}=29, P<0.044$, two-sided without

4 multiple comparisons) (Fig. 3). Furthermore, we confirmed that the behavioral differences in

5 performances between the DMN-state and DAN-state were robust to choice of ROIs

6 (Supplementary Figures 2 and 3). These results indicate that participants can maintain more

7 stable and accurate performance during DMN-state than those during DAN-state. That is, the

8 DMN-state is a behaviorally optimal state and the DAN-state is behaviorally suboptimal 9 state.
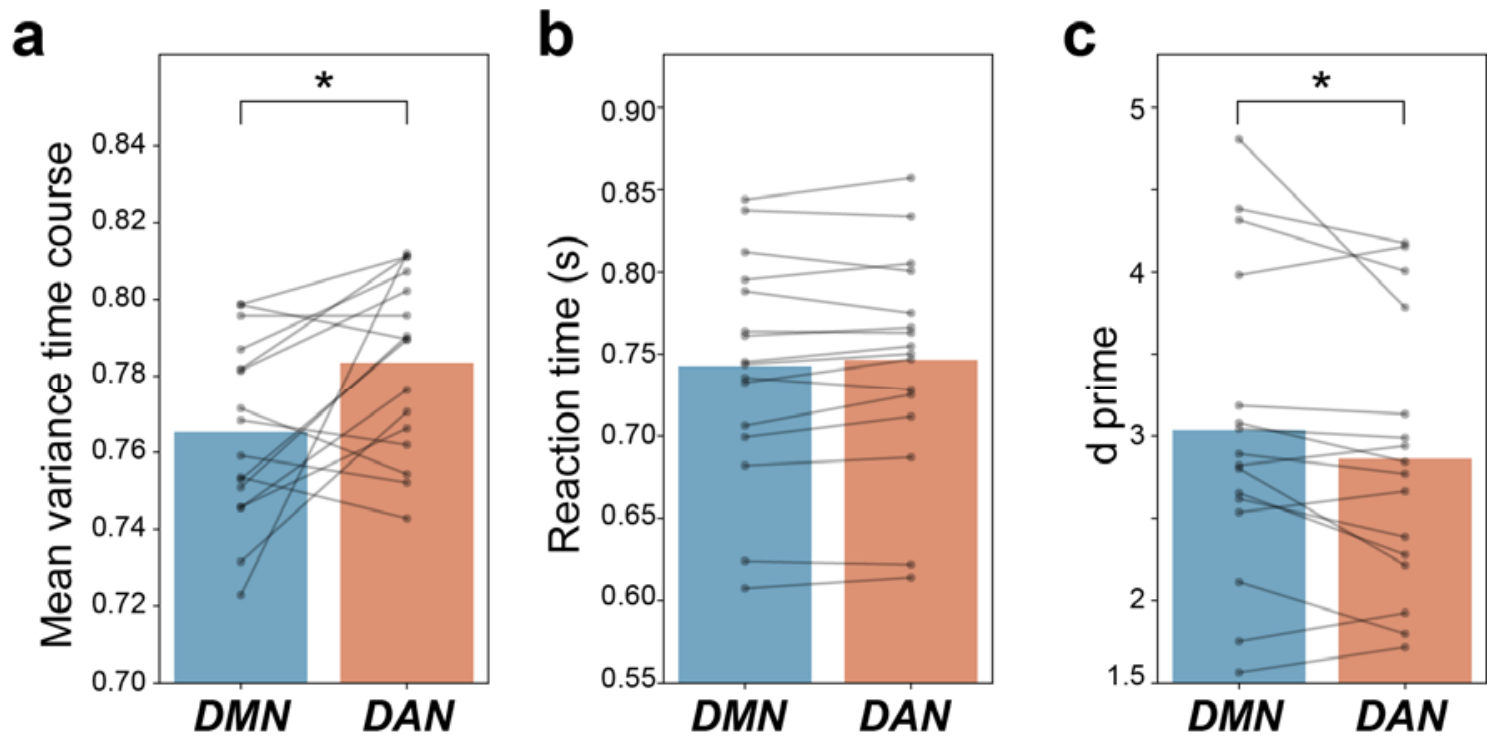

Figure 3. Behavioral performance during each state. (a) Mean variance time course. (b) Reaction time. (c) d prime. Each scatter shows each participant and line connected the same participant. DMN: default mode network; DAN: dorsal attention network. ${ }^{*} P<$ 0.05 .

Replication using an independent validation dataset. We tested whether optimal and males, ages 21-36 years, mean age $=26.4$ years) did five gradCPT runs that had a longer 
1 fMRI runs of gradCPT were gradCPT with thought-probe (see Long inter stimulus interval

2 (ISI) gradCPT dataset (Dataset 2) in the Methods section). Here, we did not use the

3 thought-probe measurement (but we did subsequently in Investigation of the influence of

4 additional cognitive and clinical factors section). We applied the identical analysis procedure

5 to this independent dataset and found consistent results with the previous experiment that the

6 DMN-state and DAN-state accounted for $49 \%$ and $45 \%$ of the total scan duration,

7 respectively (Fig.3b right). Although there appears to be 4 main local minimum states

8 (Fig.3a), the pair of brain states 2 and 3 as well as the pair of brain states 1 and 4 are different

9 only in the inclusion or exclusion of the cerebellum ROIs, respectively.

10 We confirmed that the differences in behavioral performance between during the DMN-

11 and DAN-states largely replicated in this dataset (Wilcoxon signed-rank test. Variance time

12 course: $W_{28}=57, P<0.00052$; Reaction time: $W_{28}=32, P<0.000061$; d prime: $W_{28}=73, P$

$13<0.0018$, two-sided without multiple comparisons) (Fig. 3cde). Since this dataset included

14 more subjects and more sessions than study 1 included, or because of the slower ISI, we

15 found that mean RT was also faster in the DMN-state. That is, the DMN-state is a

16 behaviorally optimal state and the DAN-state is behaviorally suboptimal state even in this

17 independent dataset. 

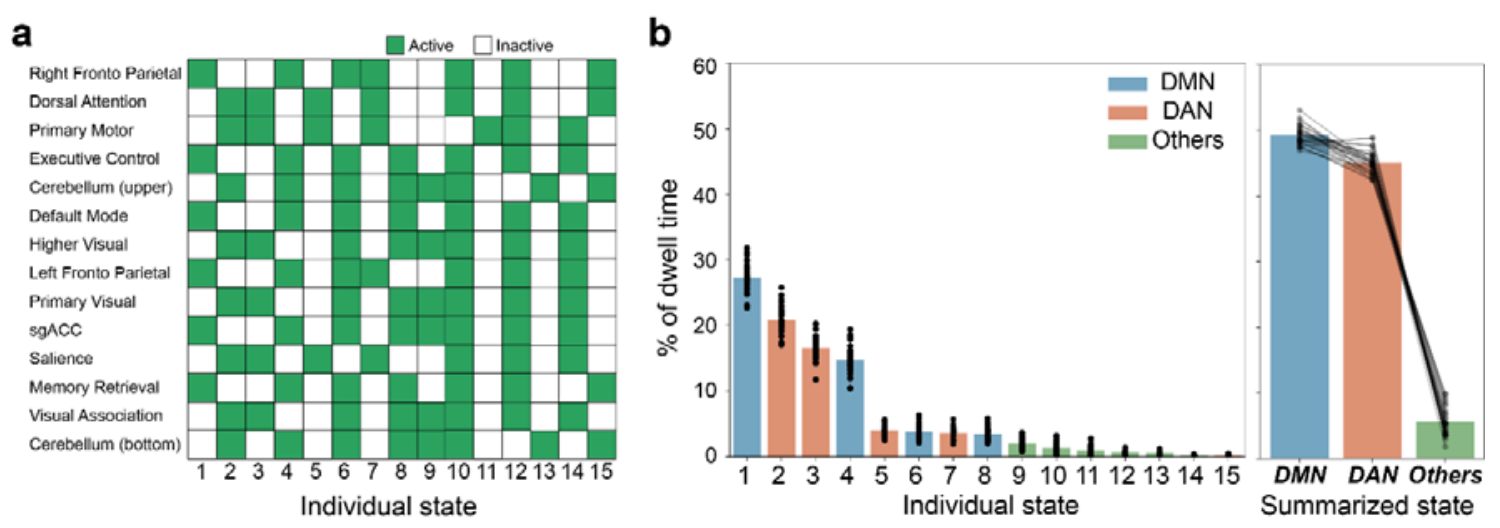
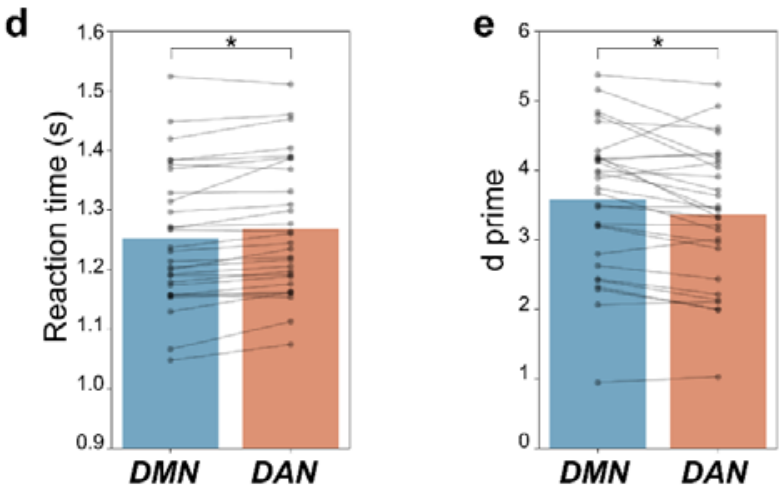

Figure 4. Replication using the independent validation dataset. (a) Stable brain states during long inter-stimulus interval gradual onset continuous performance task (gradCPT). (b) Percentage of dwell time during long inter-stimulus interval gradCPT. (c) Mean variance time course during each state. (d) Reaction time during each state. (e) d prime during each state. Each scatter shows each participant and line connected the same participant. DMN: default mode network; DAN: dorsal attention network. ${ }^{*} P<0.05$.

Investigation of the influence of additional cognitive and clinical factors. Next, we investigated how motivation, mind wandering, and ADHD affected sustained attention and the characteristics of these brain states. There are four possibilities for the impact of these factors: (1) the factor directly impact the nature of the brain state(s) (alters the brain activity pattern of brain state), (2) the factor impact the dynamics of the brain state (s) (alters the dwell time in brain state), (3) the factor impacts performance across both brain states equally, (4) the factor impacts performance differentially in one brain state. 
1 Influence of motivation. First, we investigated how reward-induced motivation affects

2 performances and brain states using a different fMRI dataset in which 16 participants (10

3 males, ages $=19-29$, mean age $=22$ years $)$ performed $3-5$ 8-min runs of the gradCPT $(13$

4 participants completed five runs, 2 completed four runs, and 1 completed three runs) with

5 performance-based rewards (see gradCPT with reward data set (Dataset 3 ) in the Methods).

6 Each 8-min task run was divided into alternating 1-min motivated and unmotivated blocks,

7 which were differentiated by a continuous color border (green for motivated; blue for

8 unmotivated). This yielded 4 min of each block-type per run. During the motivated block,

9 participants earned bonus money for correct responses and lost bonus money for mistakes

10 and during the unmotivated blocks, no money could be gained or lost. These identical reward

11 contingencies were shown to produce reliable improvements in accuracy and RT variability

12 in previous $\mathrm{study}^{34}$, thus a priori, we were certain that these performance-based rewards

13 modulated sustained attention performance.

We divided all BOLD signals from 14 ROIs into rewarded and unrewarded blocks

15 and concatenated BOLD signals from all participants for each block. We shifted the time

16 labels of the brain states backwards by 5 seconds to account for the hemodynamic response.

17 We then conducted the energy landscape analysis separately in each block and investigated

18 the stable brain states. As previously found in studies 1 and 2, two dominant brain states were

19 observed for both block types (Supplementary Figure 4). That is, DMN-state and DAN-state

20 were dominant in rewarded blocks (DMN-state: 47\%, DAN-state: 52\%) and unrewarded

21 block (DMN-state: 49\%, DAN-state: 51\%) (Fig.5a).

22 We first verified whether the relationship between brain states and behavior could be 23 replicated when using only unmotivated block data, akin to studies 1 and 2 (both without 24 reward). The unmotivated block data is not exactly same as in the first gradCPT dataset 25 because the unmotivated block data might be actively unmotivated by the presence of the 26 motivated block. However, we successfully replicated the difference in performance between 
1 DMN-state and DAN-state state (Wilcoxon signed-rank test. mean VTC: $W_{15}=5, P<$

$2 \quad 0.0012$; RT: $W_{15}=15, P<0.0062$; d prime: $W_{15}=4, P<0.00094$, two-sided without multiple

3 comparisons) (Fig.5 pale color). The results using only motivated block data are in the Figure

$4 \quad 5$ and the legend.

5 We then investigated whether there were significant interactions between brain state and 6 motivation for dwell time and behavioral performances. We found a significant interaction 7 effect for dwell time as well as d prime (Mixed effects model [interaction effect between 8 brain state and motivation]. Dwell time: $t_{60}=-2.03, P<0.047$; d prime: $t_{60}=-2.98, P<$ $9 \quad 0.0042$, two-sided without multiple comparisons) (Fig. 5). d prime was significantly better in

10 DMN-state than that in DAN-state during unmotivated block (Mixed effects model [effect of 11 motivation]. $t_{30}=-5.75, P<5.6 \times 10^{-6}$, two-sided after Bonferroni correction for two 12 comparisons [two blocks]), but not during motivated block (Mixed effects model [effect of 13 motivation]. $t_{30}=-0.23, P>0.99$, two-sided after Bonferroni correction for two comparisons 14 [two blocks]). This result indicates that motivation could partially overcome the suboptimal 15 brain state's impact on performance. On the other hand, despite the dwell time interaction, 16 there were no significant dwell time differences between motivated and unmotivated blocks 17 in either brain states (Mixed effects model [effect of motivation]. DMN-state: $t_{30}=1.91, P>$

18 0.12; DAN-state: $t_{30}=-0.95, P>0.68$, two-sided after Bonferroni correction for two 19 comparisons [two states]). 
Influence of mind wandering. Second, we investigated the effect of mind wandering using

a

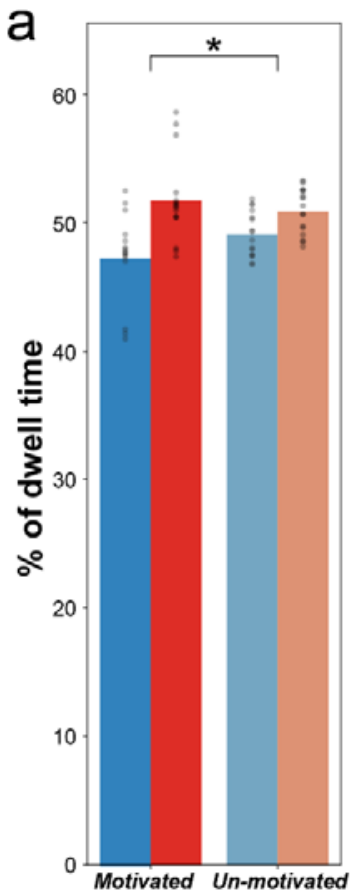

b

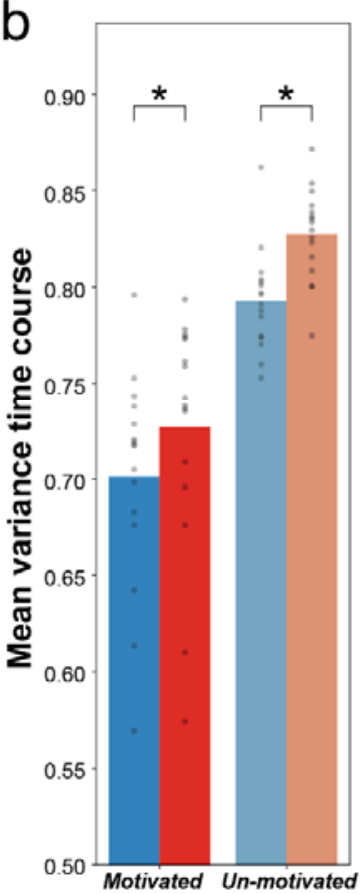

Motivated DMN DAN

C

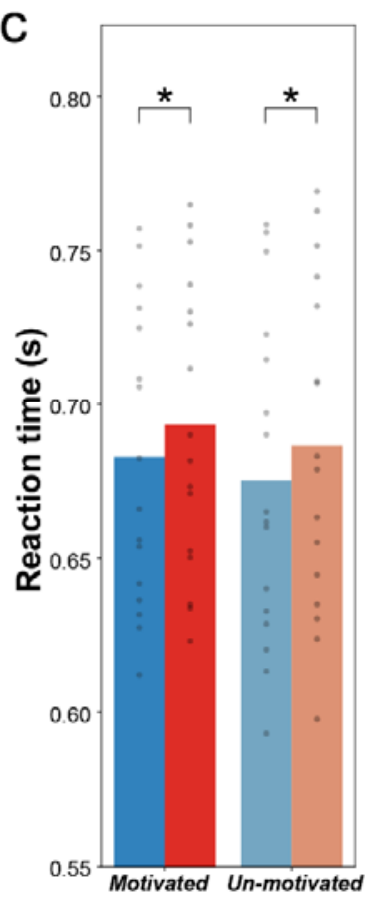

Un-motivated

DMN DAN

d

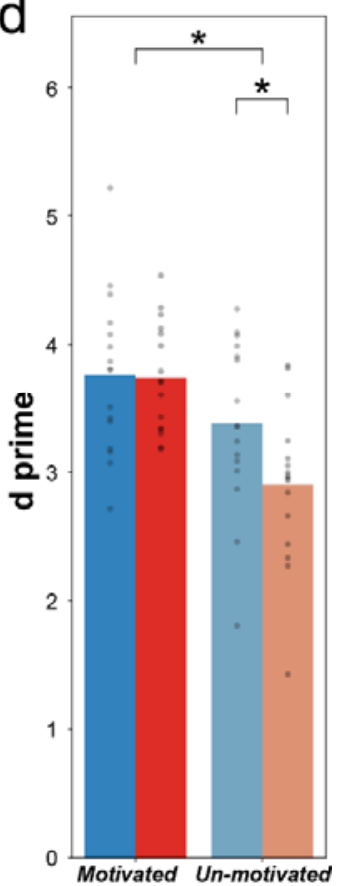

Figure 5. Effect of motivation on dwell time and behavioral performance. (a) Percentage of dwell time during each state. (b) Mean variance time course during each state. (c) Reaction time during each state. (d) d prime during each state. We successfully replicated the difference in performance between DMN-state and DAN-state state in the unmotivated block data and partially replicated in the motivated block data (Wilcoxon signed-rank test. mean VTC: $W_{15}=24, P<0.03$; RT: $W_{15}=2, P<0.00065$; d prime: $W_{15}$ $=53, P>0.43$, two-sided without multiple comparison among performances). $* P<0.05$. another fMRI dataset, collected on the same participants from study 2 (part of Dataset 2, see Long inter stimulus interval (ISI) gradCPT dataset (Dataset 2) in the Methods). This version of the gradCPT estimated subjects' self-reported mind wandering degree during gradCPT using experience sampling approach ${ }^{42} .29$ participants performed 4 gradCPT runs during fMRI, modified here to include thought-probes. Thought-probes appeared pseudorandomly every 44-60 s (three possible block durations of 44, 52, and $60 \mathrm{~s}$ ). Upon the thought-probe, a question was displayed: "To what degree was your focus just on the task or on something else?" A continuous scale appeared below the question text with far-right and far-left anchors 
1 of only task and only else, respectively. Responses were recorded on a graded scale of

2 integers (not visible to the subjects) ranging from 0 (only task) to 100 (only else). This

3 yielded 9 mind wandering degrees every $44-60$ s of per run. Following the previous study

4 using this data ${ }^{22}$, we focus on -30 -s preprobe periods to best yoke thought probe ratings to

5 behaviors. We defined each 30s period as high mind wandering (high MW) periods or low

6 mind wandering (low MW) periods by performing a median split of the 9 mind wandering

7 judgements (high MW period: judgement $\geq$ median judgement). We concatenated these

8 periods separately and analyzed these as high MW blocks and low MW blocks.

9 We divided all BOLD signals from 14 ROIs into high MW blocks and low MW

blocks and concatenated BOLD signals from all participants for each block. We shifted the

11 time labels of the brain states backwards by 5 seconds to account for the hemodynamic

12 response. We then conducted the energy landscape analysis separately in each block type and

13 investigated stable brain states. Again, we found two dominant brain states for both blocks

14 (Supplementary Figure 5). That is, DMN-state and DAN-state were dominant even in high

15 MW block (DMN-state: 49\%, DAN-state: 47\%) and low MW block (DMN-state: 49\%,

16 DAN-state: 48\%) (Fig.6a).

We then investigated whether there were significant interactions between brain state

18 and mind wandering for dwell time as well as behavioral performances. We found significant

19 interactions between brain state and mind wandering for the mean VTC (Mixed effects model

20 [interaction effect between mind wandering and brain state]. $t_{112}=-2.07, P<0.042$, two

21 sided without multiple comparisons) (Fig. 6). The difference in the mean VTC between high

22 and low mind wandering level in DAN-state was significantly larger than that in DMN-state

23 (Wilcoxon signed-rank test. $W_{28}=89, P<0.0055$, two-sided). This result indicates that when

24 participants are in the suboptimal brain state, mind wandering particularly impacts

25 performance negatively, namely increasing variability of reaction time. 


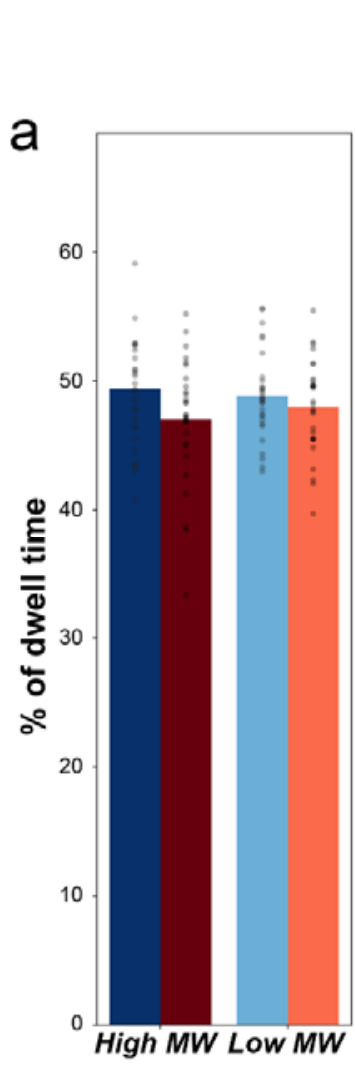

b

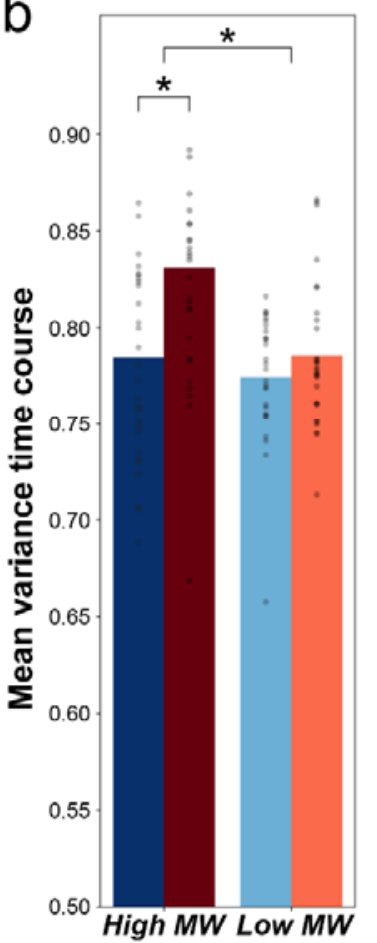

High MW

DMN DAN

C

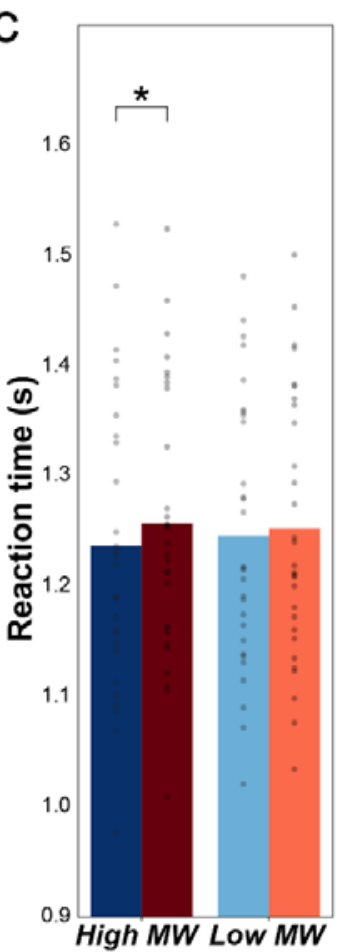

Low MW

DMN DAN

Figure 6. Effect of mind wandering on dwell time and behavioral performance. (a) Percentage of dwell time during each state. (b) Mean variance time course during each state. (c) Reaction time during each state. (d) d prime during each state. $* P<0.05$.

Influence of $A D H D$. Finally, we investigated the effect of a neuropsychiatric disorder of attention (ADHD) using another fMRI dataset consisting of 19 adult participants with ADHD (8 males, ages $18-34$ years, mean age $=24$ years $)$ who performed the gradCPT with longer inter-stimulus interval (Dataset 4) (see Long inter stimulus interval (ISI) gradCPT dataset (Dataset 2) in the Methods). We applied the identical analysis procedure to this ADHD dataset. We again found two dominant brain states (Supplementary Figure 6). That is, DMN-state and DAN-state were dominant even in ADHD patients (DMN-state: 49\%, DAN-state: 47\%) (Fig.7a).

We further verified the difference of behavioral performances between during DMN-state and DAN-state (Wilcoxon signed-rank test. Variance time course: $W_{18}=3.0, P<$ 0.0002; Reaction time: $W_{18}=10.0, P<0.00063$; d prime: $W_{18}=51.0, P>0.07$, two-sided 
1 without multiple comparisons) (Fig. 7). Although d prime was not significantly different

2 between DMN-state and DAN-state in ADHD patients, this may be because of lower d prime

3 overall in ADHD patients than that in heathy controls (DMN-state: ADHD d prime $=2.80$,

$4 \mathrm{HC}$ d prime $=3.57$; DAN-state: $\mathrm{ADHD} \mathrm{d}$ prime $=2.72, \mathrm{HC} \mathrm{d}$ prime $=3.36$ ).

5 Using study 2 (non-ADHD controls) as a comparison group, we found a significant 6 interaction between brain state and group for the dwell time (Mixed effects model

$7 \quad$ [interaction effect between brain state and group]. $t_{92}=4.54, P<1.70 \times 10^{-5}$, two-sided

8 without multiple comparisons) (Fig. 7). The dwell times of DAN-state were not significantly

9 different between ADHD and HC (Mixed effects model [effect of group]. $t_{46}=-1.09, P>$

10 0.56, two-sided after Bonferroni correction for two comparisons [two states]), however the

11 dwell time of DMN-state was significantly shorter in ADHD patients than in HC (Mixed

12 effects model [effect of group]. $t_{46}=-7.58, P<2.46 \times 10^{-9}$, two-sided after Bonferroni

13 correction for two comparisons [two states]). This result indicates that individuals with

14 ADHD spend less time in the optimal DMN-state than that did HCs, while there was no

15 significant difference in time spent the suboptimal DAN-state.
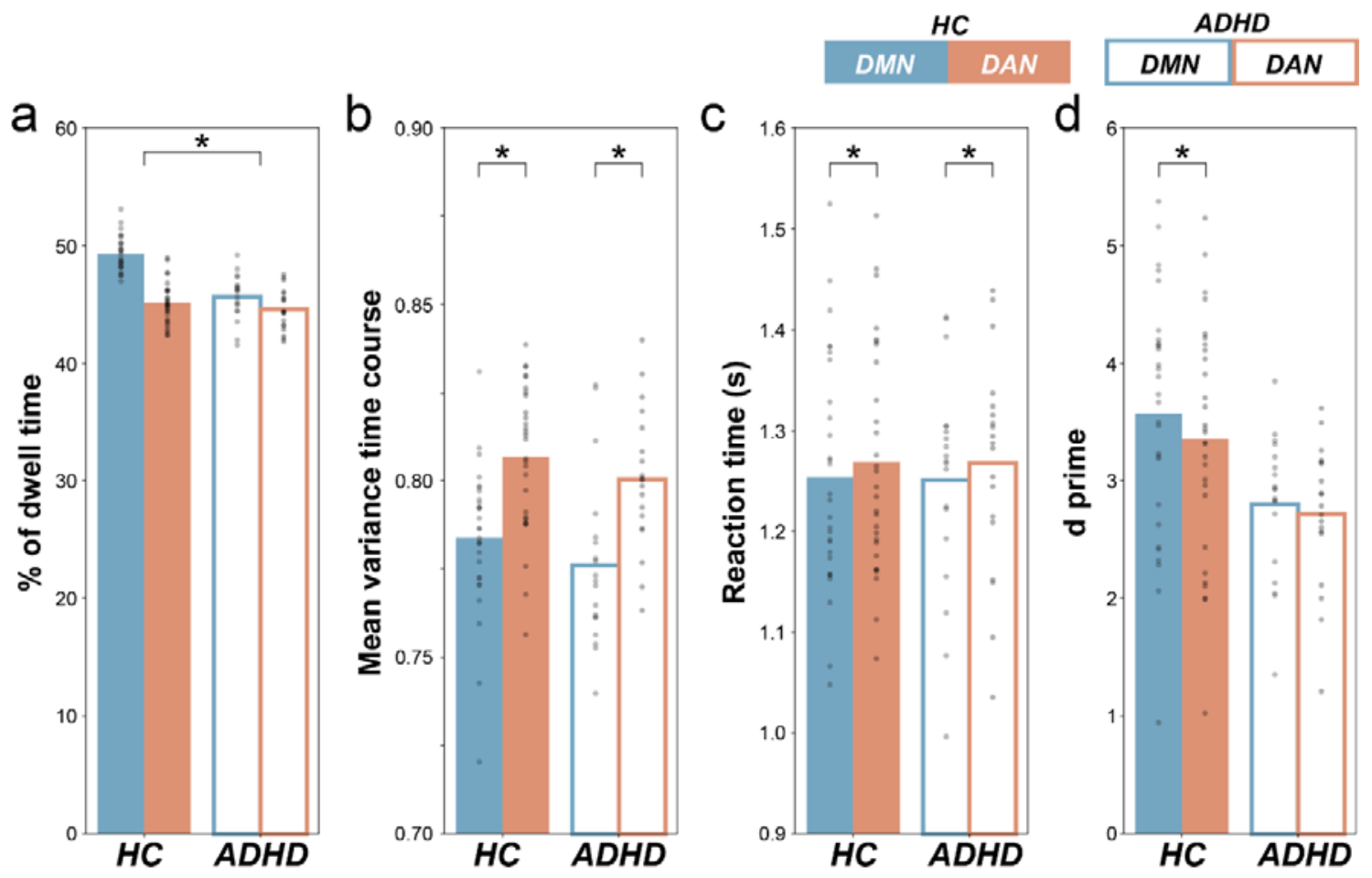


\section{Discussion}

6 In the present study, we demonstrated a systematic relationship between dynamic brain

Figure 7. Effect of ADHD on dwell time and behavioral performance. (a) Percentage of dwell time during each state. (b) Mean variance time course during each state. (c) Reaction time during each state. (d) d prime during each state. $* P<0.05$.

activity patterns across the functionally different brain systems and behavioral underpinnings of sustained attention by explaining behavior from observed brain states. This largely confirmed previous findings using behaviorally defined states such that behavioral performance (reaction time variability and accuracy) were superior during a DMN-active state compared to a DAN/SN-active state. That is, DMN-state reflected a more optimal attentional state and the DAN-state reflected a more suboptimal attentional state. We replicated our results in multiple independent datasets. We further revealed the impact of motivation, which does not change the nature and dynamics of these brain-states but serves to overcome performance decrements normally associated with the suboptimal state. Conversely, mind wandering exacerbated the negative impact on performance during the suboptimal state. Finally, we found that individuals with ADHD did alter the dynamics of the brain state by decreasing the dwell time spend in the optimal DMN-state relative to healthy controls.

Our results provided evidence for optimal and suboptimal brain states when defining state independently from behavior. This enabled us to estimate subjects' attentional states from brain activity without requiring overt responses from subjects. Since it is relatively difficult to get frequent and continuous behavior from many naturalistic tasks, as well as from some patient populations, our attentional states defined by brain activity enhance the ability to track hidden states of attention and could help better reveal neurobiological mechanisms underlying disorders of attention.

In this study, behavioral performance was consistently better during the DMN-state than during DAN-state. This is consistent with several other studies from our lab and others 
1 demonstrating DMN activity associated with stable and optimal behavior, and DAN/SN

2 associated with unstable and suboptimal behavior, when states were defined behaviorally ${ }^{21}$.

3 On the other hand, since DMN activity more typically represents "off-task" and is related to

4 mind wandering ${ }^{43}$, it has been thought that DMN activity has a negative impact on

5 performance ${ }^{17,20}$. However, previous studies showed that such relationships are highly

6 complex, such that spontaneous DMN activity can be related to both mind wandering and

7 stable "in the zone" performance ${ }^{22}$. This result indicates that there are multiple possible

8 neural mechanisms for DMN activity. Consistent with this idea, our results did not find

9 significant difference in the time occupied by DMN-state between the high mind wandering

10 blocks and the low mind wandering blocks, suggesting that the difference in the behavioral

11 performance between the DMN-state and DAN-state could be due to a largely distinct

12 mechanisms from mind wandering.

13 Many previous studies have examined differences in brain activity when behavioral

14 performance was better or worse, without considering the connectome $e^{17,20,21,24}$. Studies have

15 also examined the relationship between individual differences in the connectome between

16 functionally different brain systems and sustained attention performance, without considering

17 the brain activity ${ }^{11,19,23,24}$. However, whether and how the connectome relates to sustained

18 attention through the intermediary of their dynamic brain activity has remained unclear ${ }^{28}$. Our

19 energy landscape model clarified this relationship, as it indicates that two stable functional

20 brain systems' activity patterns with different attentional performance frequently occur under

21 the constraints of connectome.

22 On the one hand, our results showed that a positive modulator of sustained attention

23 (motivation) partially overcomes the negative effect of the suboptimal DAN-state while a

24 negative modulator of sustained attention (mind wandering) worsens the negative effect in

25 this suboptimal state. This suggest that within-subject fluctuations in performance are more

26 malleable during this suboptimal state. Interestingly, our results showed that when in this 
1 suboptimal brain state, motivation improves d-prime while mind wandering increase RT

2 variability. This might suggest that mind wandering and motivation affect cognitive

3 performance during this state via different neural mechanisms (e.g. effect mainly on visual

4 system or on motor system). This is consistent with the interpretation that intrinsic

5 fluctuations of performance are largely distinct from other cognitive mechanisms such as

6 mind wandering and motivation ${ }^{22,34}$. On the other hand, individuals with ADHD spent less

7 time in the optimal brain state than healthy controls, while the relationship between brain

8 states and behavioral performances was comparable across group. These results indicate that

9 within-subject level modulators (motivation and mind wandering) impact the optimality of behavior in the suboptimal brain state, rather than characteristics of the brain state itself. In contrast, between-subject level differences (ADHD vs HC) directly impact the optimal brain state character, namely its frequency. This may indicate that the optimal brain state is less susceptible to positive and negative effects at the intraindividual level, but can be related to interindividual differences in attention ability. As the so-called "in the zone" state may reflect automated information processing ${ }^{44}$ and loss awareness of all other things except for task in progress, the optimal brain state identified in this study may have captured this experience.

The energy landscape created in this study is a type of generative model. Generative models enables us to simulate a transition of brain state when brain network changes (e.g.

19 simulation of drug or connectivity neurofeedback effect) or activity of specific brain regions 20 are inhibited or activated (e.g. simulation of brain stimulation) $)^{26,27,45}$. Therefore, in future studies it may be possible to determine optimal targets for neurofeedback and brain stimulation to efficiently remediate and improve sustained attention ability by using our $23 \operatorname{model}^{27,45-47}$.

24 One limitation of our study is the assumption that brain networks themselves are stable.

25 Recent work suggested a relationship between dynamic functional connectivity and 26 attentional states, thus networks themselves may reconfigure with attentional fluctuations ${ }^{18,25}$. 
1 Novel unsupervised learning techniques, based on Bayesian switching linear dynamical

2 systems (BSDS), provides an integrated framework for identifying latent brain states and

3 dynamic brain connectivity during cognitive tasks ${ }^{48}$. In the future, such advanced techniques

4 could be used to investigate brain states that take into account dynamic functional

5 connectivity and relationship between individual brain state and individual behavioral

6 performance.

7 In summary, our study is the first to provide evidence for two attentional states, a

8 behaviorally optimal and suboptimal states, from the viewpoint of brain activity. Additionally,

9 our study shows that activity patterns across functionally different brain systems could be the

10 link between prior relationships between functional connectivity (connectome) and sustained

11 attention. Our results indicate that a within-subject level positive modulator (motivation) and

12 a negative modulator (mind wandering) impacted task performance within these brain states,

13 but not the character (composition and frequency) of the brain states themselves. Furthermore,

14 our results suggest that behavior was more susceptible to cognitive modulators of attention

15 when in the suboptimal brain state. On the other hand, our results suggest that while the

16 composition of stable brain states were not different across a between-subject level factor

17 (individual with ADHD vs healthy controls), the time spent in the optimal brain state was

18 shorter in ADHD patients than in healthy controls.

19 We believe this approach has wide ranging implications for neurocognitive and clinical 20 models of attention, and can set a new methodological and theoretical trajectory for a wealth 21 of future studies. 


\section{Methods}

2 gradCPT data set (Dataset 1). Participants. Sixteen participants (6 males, ages 18-34 years, mean age =

324.1 years) performed the gradual onset continuous performance task (gradCPT) during functional

4 magnetic resonance imaging (fMRI). The data used in this study and portions of the methods have been

5 published $^{21}$, but the current analyses and results reported have not been published elsewhere. To identify

6 network level functional region of interests (ROIs), a 6-min resting state fMRI was collected and submitted

7 to dictionary learning (details below). All participants were right handed, with normal or

8 corrected-to-normal vision and no reported history of major medical illness, head trauma, neurological, or

9 psychiatric disorder. The study was approved by the VA Boston Healthcare System IRB, and written

10 consent was obtained from all participants.

11 Task Paradigm and Presentation. The gradCPT contained 10 round, grayscale photographs of 12 mountain scenes and 10 of city scenes. These scenes were randomly presented with $10 \%$ mountain and $1390 \%$ city, without allowing the identical scene to repeat on consecutive trials. Scene images gradually 14 transitioned from one to the next, using a linear pixel-by-pixel interpolation, with each transition occurring 15 in $800 \mathrm{~ms}$. Images were projected to participants through a MR compatible goggle system (VisuaStim 16 Digital, Resonance Technology Inc.), and subtended a radius of $2.2^{\circ}$ of visual angle. Participants were 17 instructed to press a button for each city scene, and withhold responses to mountain scenes. Response 18 accuracy was emphasized without reference to speed. However, given that the next stimulus would replace the current stimulus in $800 \mathrm{~ms}$, a response deadline was implicit in the task.

Behavioral analysis: Reaction time. Reaction times (RT) were calculated relative to the beginning of each image transition, such that an RT of $800 \mathrm{~ms}$ indicates a button press at the moment image $\mathrm{n}$ was $100 \%$ coherent and not mixed with other images. A shorter RT indicates that the current scene was still in the process of transitioning from the previous, and a longer RT indicates that the current scene was in the process of transitioning to the subsequent scene. So, for example, an RT of 720 ms would be at the moment of $90 \%$ image $\mathrm{n}$ and $10 \%$ image $\mathrm{n}-1$, and so forth. On rare trials with highly deviant RTs (before $70 \%$ coherence of image $\mathrm{n}$ and after $40 \%$ coherence of image $\mathrm{n}+1$ ) or multiple button presses, an iterative algorithm maximized correct responses as follows. The algorithm first assigned unambiguous correct responses, leaving few ambiguous button presses (presses before 70\% coherence of the current scene and after $40 \%$ coherence of the following scene or multiple presses occurred on $<5 \%$ of trials). Second, ambiguous presses were assigned to an adjacent trial if 1 of the 2 had no response. If both adjacent trials had no response, the press was assigned to the closest trial, unless one was a no-go target, in which case subjects were given the benefit of the doubt that they correctly omitted. Finally, if there were multiple presses that could be assigned to any 1 trial, the fastest response was selected. Slight variations to this algorithm yielded highly similar results, as most button presses showed a 1-1 correspondence with presented images.

Behavioral analysis: Variance time course. Beyond mean RT and error rates, we were particularly interested in trial-to-trial variation in RT, which we assessed via a novel within subject analysis that we called the variance time course (VTC) ${ }^{21}$. VTCs were computed from the -500 correct responses in each run (following z-transformation of RTs within-subject to normalize the scale of the VTC), where the 
We reasoned that deviant RTs, whether fast or slow, represented reduced attention to the task as follows: extremely fast RTs often indicate premature responding and inattention to the potential need for response inhibition ${ }^{49}$, while extremely slow RTs might indicate reduced attention to or inefficient processing of the ongoing stream of visual stimuli, requiring more time to accurately discriminate scenes ${ }^{17}$. Values for trials without responses (omission errors and correct trials) were interpolated linearly, such that the missing values were linearly estimated from RTs of the 2 surrounding trials. A smoothed VTC was computed using a Gaussian kernel of 9 trials ( $\sim \mathrm{s}$ ) full-width at half-maximum (FWHM), thus integrating information from the surrounding 20 trials, or $16 \mathrm{~s}$, via a weighted average. This choice was based on prior work linking fluctuations around this frequency to attentional impairments ${ }^{50}$.

MRI Acquisition. Scanning was performed on a 3T Siemens MAGNETOM Trio system equipped with a 12-channel head coil, at the VA Boston Neuroimaging Research Center. Functional runs included 248 (gradCPT) or 188 (resting state) whole-brain volumes acquired using an echo-planar imaging sequence with the following parameters: repetition time $(\mathrm{TR})=2000 \mathrm{~ms}$, echo time $(\mathrm{TE})=30 \mathrm{~ms}$, flip angle $=90^{\circ}$, acquisition matrix $=64 \times 64$, in-plane resolution $=3.0 \mathrm{~mm}^{2}, 33$ oblique slices, slice thickness $=3,0.75 \mathrm{~mm}$ gap. MPRAGE parameters were as follows: $\mathrm{TE}=3.32, \mathrm{TR}=2530 \mathrm{~ms}$, flip angle $=7^{\circ}$, acquisition matrix $=256 \times 256$, in-plane resolution $=1.0 \mathrm{~mm}^{2}, 176$ sagittal slices, slice thickness $=1.0$ $\mathrm{mm}$.

fMRI analysis: Preprocessing of fMRI. We performed preprocessing of the fMRI data using FMRIPREP version 1.3.0 ${ }^{51}$. Preprocessing steps included realignment, coregistration, segmentation of T1-weighted structural images, normalization to Montreal Neurological Institute (MNI) space. For more details of the pipeline, see http://fmriprep.readthedocs.io/en/latest/workflows.html.

Parcellation of brain regions: Dictionary learning. We identified functionally different brain systems to use as functional ROIs from the whole brain by applying dictionary learning to resting state fMRI data ${ }^{36-38}$. We first concatenated all participants' resting state fMRI and then applied dictionary learning implemented in Nilearn ${ }^{52}$. Dictionary learning is a sparse based decomposition method for extracting spatial maps. We set the number of components as 20 . We used spatial smoothing with an isotropic Gaussian kernel of $6 \mathrm{~mm}$ full-width at half-maximum. A temporal bandpass filter was applied to the time series using a first-order Butterworth filter with a pass band between $0.01 \mathrm{~Hz}$ and $0.08 \mathrm{~Hz}$ to restrict the analysis to low-frequency fluctuations, which are characteristic of resting state fMRI BOLD activity $^{53}$. The BOLD signal time courses were extracted from these 20 ROIs. We visually inspected 5 ROIs which were considered as noise and an auditory related ROI which were not related to our current task and excluded from our current analysis. We finally used 14 ROIs for all analysis (Fig. 1a).

Physiological noise regression: Physiological noise regressors were extracted by applying $\mathrm{CompCor}^{54}$. Principal components were estimated for the anatomical CompCor (aCompCor). A mask to exclude signals with a cortical origin was obtained by eroding the brain mask and ensuring that it contained subcortical structures only. Six aCompCor components were calculated within the intersection of the subcortical mask and union of the CSF and WM masks calculated in T1-weighted image space after their projection to the native space of functional images in each session. Furthermore, to isolate the effect of each trial type (commission error, correct omission, correct commission, omission error) as well as trial-to-trial RT, we included mean evoked response for each trial type and trial-to-trial RT. We estimated 
BOLD response time courses of each event type by using hemodynamic_models function implemented in Nistat (https://nistats.github.io/). To remove several sources of spurious variance, we used linear regression with eighteen regression parameters, including six motion parameters, average signal over the whole brain, six aCompCor components, estimated BOLD response time course of each event type, and estimated BOLD response time course of trial-to-trial RT.

Pairwise maximum entropy model. We fitted the pairwise Maximum entropy model (MEM) to the preprocessed BOLD signals as follows in the same manner as that employed in the previous studies $^{29-33,39}$. We used open toolbox so called Energy Landscape Analysis Toolkit (ELAT) (https://sites.google.com/site/ezakitakahiro/software). Since this method cannot be applied to relatively large amount of data ${ }^{30}$, to achieve a high accuracy of fitting we had to reduce the number of ROIs to 14 ROIs of 20 ROIs excluding 6 ROIs which seemed to be visually noise or auditory related components. For each ROI, we first binarized the obtained fMRI signals with a threshold that was defined as the time-averaged activity of the same ROI. We then concatenated BOLD signals from all participants for each ROI. Previous studies suggest that binarization does not eliminate important information contained in originally continuous brain signals ${ }^{31,32,39}$. In this method, the binarized activity $\sigma_{i}^{t}$ at ROI $i$ and discrete time $t$ is either active or inactive $(+1$ or 0$)$. The activity pattern at time $t$ is described by $V^{t}=\left[\sigma_{1}^{t}, \sigma_{2}^{t}, \ldots, \sigma_{N}^{t}\right]^{\mathrm{T}}$ where $N(=14)$ is the number of the ROIs. The $k$ th brain activity pattern is described by $V_{k}\left(k=1,2, \ldots, 2^{N}\right)$. Specifically, when the empirical activation of ROI $i,\left\langle\sigma_{i}\right\rangle$, and the empirical pairwise activation of ROIs $i$ and $j,\left\langle\sigma_{i} \sigma_{j}\right\rangle$, are estimated from the data, the probability distribution of the $k$ th brain activity pattern with the largest entropy is the Boltzmann distribution ${ }^{55}$. Here, $\left\langle\sigma_{i}\right\rangle$ is equal to $(1 / T) \sum_{t=1}^{T} \sigma_{i}^{t}$ and $\left\langle\sigma_{i} \sigma_{j}\right\rangle$ is equal to $(1 / T) \sum_{t=1}^{T} \sigma_{i}^{t} \sigma_{j}^{t}$, where $T$ is the number of volume. That is, $P\left(V_{k}\right)=e^{E\left(V_{k}\right)} / \sum_{l=1}^{2^{N}} e^{-E\left(V_{l}\right)}$, where $E\left(V_{k}\right)$ is the energy of activity pattern $V_{k}$ and is given by $E\left(V_{k}\right)=-\sum_{i=1}^{N} h_{i} \sigma_{i}\left(V_{k}\right)-\frac{1}{2} \sum_{i=1}^{N} \sum_{j=1, j \neq i}^{N} J_{i j} \sigma_{i}\left(V_{k}\right) \sigma_{j}\left(V_{k}\right)$. Here, $\sigma_{i}\left(V_{k}\right)$ represents the binarized activity $(+1$ or 0$)$ at region $i$ under activity pattern $V_{k}$. Technically, $h_{i}$ and $J_{i j}$ in the Boltzmann distribution were adjusted until the model-based mean ROI activity $\left\langle\sigma_{i}\right\rangle_{m}=\sum_{l=1}^{2^{N}} \sigma_{i}\left(V_{l}\right) P\left(V_{l}\right)$ and model-based mean pairwise interaction $\left\langle\sigma_{i} \sigma_{j}\right\rangle_{m}=\sum_{l=1}^{2^{N}} \sigma_{i}\left(V_{l}\right) \sigma_{j}\left(V_{l}\right) P\left(V_{l}\right)$ were approximately equal to the empirically obtained $\left\langle\sigma_{i}\right\rangle$ and $\left\langle\sigma_{i} \sigma_{j}\right\rangle$.

Energy landscape analysis. We calculated the energy landscape as done in the previous studies $^{29,30,32,33}$. The energy landscape is defined as a network of brain activity patterns $V_{k}$ with the corresponding energy $E\left(V_{k}\right)$. Two activity patterns are regarded as adjacent in the network if and only if they take the opposite binary activity at just one brain region. We first exhaustively searched for local energy minimums, whose energy values are smaller than those of all the $N$ adjacent patterns. We then summarized the all brain activity patterns into local minimum brain states. We first selected a starting brain activity pattern $i$ among the $2^{N}$ brain activity patterns. Then, if any of its neighbor patterns has a smaller value of energy than pattern $i$, we moved to the neighbor pattern with the smallest energy value. Otherwise, we did not move, which implied that pattern $i$ was a local minimum. We repeated this procedure until arrived at a local minimum. The starting pattern $i$ was regarded to belong to the local minimum that was finally reached. We estimated the corresponding local minimum for all brain activity patterns.

Brain behavior relationship analysis. By regarding local minimum brain activity patterns as brain states, all participants have a brain state transition and behavioral time series (Fig. 1b). Thus, we can 
calculate behavioral performance during each brain state. We shifted the time labels of the brain state time series backwards by $5 \mathrm{~s}$ to account for the hemodynamic response. In addition to RT and VTC, we calculated performance accuracy (d prime). d prime was calculated as $z$ (hit rate) $-z$ (false alarm rate) during each brain state. Here $\mathrm{z}$ is normal probability density function in $\mathrm{SciPy}^{56}$.

Long inter stimulus interval (ISI) gradCPT dataset (Dataset 2). Participants. 29 participants (13 males, ages 21-36 years, mean age $=26.4$ years) performed the long inter-stimulus interval (ISI) gradCPT $(1300$ ms vs. $800 \mathrm{~ms}$ per image) during fMRI. Subjects completed the following sequence of runs with short breaks separating each (lasting a total of 1.5-2 h): one multi-echo T1-weighted run, one resting-state fMRI run, one long ISI gradCPT run followed by four long ISI gradCPT runs with intermittent thought-probes. The data used in this study and portions of the methods have been published ${ }^{22}$, but the current analyses and results reported have not been published elsewhere. Subjects were screened by phone and at an initial visit before the day of neuroimaging, where subjects were also trained on performing the long ISI gradCPT. Exclusion criteria were as follows: current mood, psychotic, anxiety (excluding simple phobias) or attention-deficit/hyperactivity disorder, current use of psychotropic medication, full-scale IQ less than 80, neurological disorders, sensorimotor handicaps, current alcohol or substance abuse/dependence, and claustrophobia. Furthermore, 19 ADHD patients (8 males, ages 18-34 years, mean age $=24$ years) also performed the long ISI gradCPT during fMRI data collection (Dataset 4). We used one long ISI gradCPT run in conjunction with four long ISI gradCPT runs with thought-probes for replication analysis but used only the four long ISI gradCPT runs with thought-probes for investigating mind wandering effect.

Task Paradigm and Presentation. In four long ISI gradCPT runs with thought-probes, participants performed the gradCPT, modified here to include thought-probes. The following script was used during training on a computer (outside the scanner) to instruct participants in how to respond to the thought-probes: Thought-probes appeared pseudo-randomly every 44-60 s (three possible block durations of 44, 52, and $60 \mathrm{~s}$ ). Rather than gradually transitioning into another scene image, the last scene before the thought-probe faded into a scrambled image (to give subjects a similar amount of time to respond as in other trials). Upon the thought-probe, a question was displayed: "To what degree was your focus just on the task or on something else?" A continuous scale appeared below the question text with far-right and far-left anchors of only task and only else, respectively. Subjects pressed buttons with their middle and ring fingers to move the scale left and right, respectively, and with their thumb to enter their response. Responses were recorded on a graded scale of integers (not visible to the subjects) ranging from 0 (only task) to 100 (only else). A second self-paced question screen about meta-awareness of task-related focus (“To what degree were you aware of where your focus was?") appeared after the thought-probe, but responses for this second question were not included in the present analyses. The gradCPT immediately resumed after subjects entered their question responses (except for the last thought-probe in the run). Scanning was manually stopped after each gradCPT thought-probe run.

MRI Acquisition. Functional and anatomical MRIs were acquired on the 3T Siemens CONNECTOM scanner with a custom-made 64-channel phased array head coil, housed at the Athinoula A. Martinos Center for Biomedical Imaging. The T2*-weighted whole-brain fMRI runs were performed with multiband, echo-planar imaging (simultaneous multislice factor of 4) and the following parameters: 
repetition time (TR), $1.08 \mathrm{~s}$; echo time (TE), $30 \mathrm{~ms}$; flip angle, 60 ; field of view (FoV), $110 \mathrm{~mm} 2 ; 68$ transverse slices; $2 \mathrm{~mm}$ isotropic voxels. The T1-weighted scan parameters were as follows: TR, 2,530 ms; TE, $1.15 \mathrm{~ms}$; inversion time (TI), 1,100 ms; flip angle, 7; FoV, $256 \mathrm{~mm} 2 ; 1 \mathrm{~mm}$ isotropic voxels.

gradCPT with reward data set (Dataset 3). Participants. Sixteen participants (10 males, ages = 19-29, mean age $=22$ years $)$ completed 3-5 8-min runs of the gradCPT (13 participants completed five runs, 2 completed four runs, and 1 completed three runs) during fMRI. The data used in this study and portions of the methods have been published ${ }^{34}$, but the current analyses and results reported have not been published elsewhere. Fourteen participants were right-handed and all were considered healthy, had normal or corrected-to-normal vision, and no reported history of major illness, head trauma, or neurological/psychiatric disorders. All were screened to confirm no metallic implants or history of claustrophobia. Drug/medication use was not explicitly assessed. The study protocol was approved by the VA Boston Healthcare System Institutional Review Board, and all participants gave written informed consent

Task Paradigm and Presentation. In the gradCPT with reward data set, each 8-min task run was divided into alternating 1-min rewarded and unrewarded blocks, which were differentiated by a continuous color border (green for rewarded; blue for unrewarded). To have the background colors be more intuitive and avoid confusion, "green" was chosen for rewarded blocks in all participants rather than counterbalancing green and blue colors. This yielded 4 min of each block-type per run. Similar to our previous $s \mathrm{udy}^{35}$, participants earned $\$ 0.01$ for correctly pressing to city scenes and $\$ 0.10$ for correctly withholding a response to mountain scenes during rewarded blocks. However, if a participant failed to press to a city scene, they would lose $\$ 0.01$, and if a participant incorrectly pressed to a mountain scene they would lose \$0.10. During the unrewarded blocks, no money could be gained or lost. These identical reward contingencies were shown to produce reliable improvements in accuracy and RT variability in our recent study with 54 participants ${ }^{35}$, and in previously published work with this data ${ }^{34}$, thus a priori, we were certain the payoff matrix successfully modulated sustained attention performance.

MRI Acquisition. Scanning was performed on a 3T Siemens MAGNETOM Trio system equipped with a 32-channel head coil at the VA Boston Neuroimaging Research Center for Veterans (NeRVe). Each gradCPT functional run included 248 whole-brain volumes acquired using an echo-planar imaging sequence with the following parameters: $\mathrm{TR}=2000 \mathrm{~ms}$, $\mathrm{TE}=30 \mathrm{~ms}$, flip angle $=90^{\circ}$, acquisition matrix $=$ $64 \times 64$, in-plane resolution $=3.0 \times 3.0 \mathrm{~mm} 2,33$ oblique slices aligned to the anterior and posterior commissures, slice thickness $=3 \mathrm{~mm}$ with a $0.75 \mathrm{~mm}$ gap. MP-RAGE sequence parameters were as follows: $\mathrm{TE}=3.32 \mathrm{~ms}, \mathrm{TR}=2530 \mathrm{~ms}$, flip angle $=7^{\circ}$, acquisition matrix $=256 \times 256$, in-plane resolution $=$ $1.0 \mathrm{~mm} 2,176$ sagittal slices, slice thickness $=1.0 \mathrm{~mm}$.

Code availability. All codes used in the analyses are available from the authors on request.

Acknowledgements. This research was supported by the TOYOBO Biotechnology Foundation to AY and 
1 a Merit Review Award from the Department of Veterans Affairs Clinical Sciences Research and

2 Development (I01CX001653) to ME.

3

4 Author contributions. M.E., E.V. and A.K. designed the study and recruited participants for the study,

5 collected their clinical and imaging data. A.Y. performed data preprocessing and analysis; A.Y., D.R., and 6 M.E. primarily wrote the manuscript.

8 Competing financial interests. The authors declare no competing financial interests. 
1 Fiebelkorn, I. C., Pinsk, M. A. \& Kastner, S. A Dynamic Interplay within the Frontoparietal Network Underlies Rhythmic Spatial Attention. Neuron 99, 842-853 e848, doi:10.1016/j.neuron.2018.07.038 (2018).

2 Helfrich, R. F. et al. Neural Mechanisms of Sustained Attention Are Rhythmic. Neuron 99, 854-865 e855, doi:10.1016/j.neuron.2018.07.032 (2018).

3 Fiebelkorn, I. C. \& Kastner, S. A Rhythmic Theory of Attention. Trends in cognitive sciences 23, 87-101, doi:10.1016/j.tics.2018.11.009 (2019).

4 Mackworth, N. H. The Breakdown of Vigilance during Prolonged Visual Search. Quarterly Journal of Experimental Psychology 1, 6-21, doi:10.1080/17470214808416738 (1948).

5 Chun, M. M., Golomb, J. D. \& Turk-Browne, N. B. A taxonomy of external and internal attention. Annu Rev Psychol 62, 73-101, doi:10.1146/annurev.psych.093008.100427 (2011).

6 Fortenbaugh, F. C., DeGutis, J. \& Esterman, M. Recent theoretical, neural, and clinical advances in sustained attention research. Annals of the New York Academy of Sciences 1396, 70-91, doi:10.1111/nyas.13318 (2017).

7 Fortenbaugh, F. C., Rothlein, D., McGlinchey, R., DeGutis, J. \& Esterman, M. Tracking behavioral and neural fluctuations during sustained attention: A robust replication and extension. NeuroImage 171, 148-164, doi:10.1016/j.neuroimage.2018.01.002 (2018).

8 Huang-Pollock, C. L., Karalunas, S. L., Tam, H. \& Moore, A. N. Evaluating vigilance deficits in ADHD: a meta-analysis of CPT performance. $J$ Abnorm Psychol 121, 360-371, doi:10.1037/a0027205 (2012).

9 Marchetta, N. D., Hurks, P. P., De Sonneville, L. M., Krabbendam, L. \& Jolles, J. Sustained and focused attention deficits in adult ADHD. Journal of attention disorders 11, 664-676, doi:10.1177/1087054707305108 (2008).

10 Rosenberg, M. D., Finn, E. S., Scheinost, D., Constable, R. T. \& Chun, M. M. Characterizing Attention with Predictive Network Models. Trends in cognitive sciences 21, 290-302, doi:10.1016/j.tics.2017.01.011 (2017).

11 Rosenberg, M. D. et al. A neuromarker of sustained attention from whole-brain functional connectivity. Nature neuroscience 19, 165-171, doi:10.1038/nn.4179 (2016).

12 Lawrence, N. S., Ross, T. J., Hoffmann, R., Garavan, H. \& Stein, E. A. Multiple neuronal networks mediate sustained attention. Journal of cognitive neuroscience 15, 1028-1038, doi:10.1162/089892903770007416 (2003).

13 Kessler, D., Angstadt, M. \& Sripada, C. Growth Charting of Brain Connectivity Networks and the Identification of Attention Impairment in Youth. JAMA psychiatry 73, 481-489, doi:10.1001/jamapsychiatry.2016.0088 (2016). 
14 Hilti, C. C. et al. Evidence for a cognitive control network for goal-directed attention in simple sustained attention. Brain Cogn 81, 193-202, doi:10.1016/j.bandc.2012.10.013 (2013).

15 Drummond, S. P. et al. The neural basis of the psychomotor vigilance task. Sleep 28, 1059-1068 (2005).

16 Langner, R. \& Eickhoff, S. B. Sustaining attention to simple tasks: a meta-analytic review of the neural mechanisms of vigilant attention. Psychol Bull 139, 870-900, doi:10.1037/a0030694 (2013).

17 Weissman, D. H., Roberts, K. C., Visscher, K. M. \& Woldorff, M. G. The neural bases of momentary lapses in attention. Nature neuroscience 9, 971-978, doi:10.1038/nn1727 (2006).

18 Kucyi, A., Hove, M. J., Esterman, M., Hutchison, R. M. \& Valera, E. M. Dynamic Brain Network Correlates of Spontaneous Fluctuations in Attention. Cerebral cortex 27, 1831-1840, doi:10.1093/cercor/bhw029 (2017).

19 Kelly, A. M., Uddin, L. Q., Biswal, B. B., Castellanos, F. X. \& Milham, M. P. Competition between functional brain networks mediates behavioral variability. NeuroImage 39, 527-537, doi:10.1016/j.neuroimage.2007.08.008 (2008).

20 Hinds, O. et al. Roles of default-mode network and supplementary motor area in human vigilance performance: evidence from real-time fMRI. $J$ Neurophysiol 109, 1250-1258, doi:10.1152/jn.00533.2011 (2013).

21 Esterman, M., Noonan, S. K., Rosenberg, M. \& Degutis, J. In the zone or zoning out? Tracking behavioral and neural fluctuations during sustained attention. Cerebral cortex 23, 2712-2723, doi:10.1093/cercor/bhs261 (2013).

22 Kucyi, A., Esterman, M., Riley, C. S. \& Valera, E. M. Spontaneous default network activity reflects behavioral variability independent of mind-wandering. Proceedings of the National Academy of Sciences of the United States of America 113, 13899-13904, doi:10.1073/pnas.1611743113 (2016).

23 Rothlein, D., DeGutis, J. \& Esterman, M. Attentional Fluctuations Influence the Neural Fidelity and Connectivity of Stimulus Representations. Journal of cognitive neuroscience 30, 1209-1228, doi:10.1162/jocn_a_01306 (2018).

24 Thompson, G. J. et al. Short-time windows of correlation between large-scale functional brain networks predict vigilance intraindividually and interindividually. Hum Brain Mapp 34, 3280-3298, doi:10.1002/hbm.22140 (2013).

25 Fong, A. H. C. et al. Dynamic functional connectivity during task performance and rest predicts individual differences in attention across studies. NeuroImage 188, 14-25, doi:10.1016/j.neuroimage.2018.11.057 (2019).

26 Rosenberg, M. D. et al. Methylphenidate Modulates Functional Network Connectivity to Enhance Attention. The Journal of neuroscience : the official journal of the Society for Neuroscience 36, 9547-9557, doi:10.1523/JNEUROSCI.1746-16.2016 (2016). 
27 Yamashita, A., Hayasaka, S., Kawato, M. \& Imamizu, H. Connectivity Neurofeedback Training Can Differentially Change Functional Connectivity and Cognitive Performance. Cerebral cortex 27, 4960-4970, doi:10.1093/cercor/bhx177 (2017).

28 Mill, R. D., Ito, T. \& Cole, M. W. From connectome to cognition: The search for mechanism in human functional brain networks. NeuroImage 160, 124-139, doi:10.1016/j.neuroimage.2017.01.060 (2017).

29 Ezaki, T., Sakaki, M., Watanabe, T. \& Masuda, N. Age-related changes in the ease of dynamical transitions in human brain activity. Hum Brain Mapp 39, 2673-2688, doi:10.1002/hbm.24033 (2018).

30 Ezaki, T., Watanabe, T., Ohzeki, M. \& Masuda, N. Energy landscape analysis of neuroimaging data. Philos Trans A Math Phys Eng Sci 375, doi:10.1098/rsta.2016.0287 (2017).

31 Watanabe, T. et al. Energy landscapes of resting-state brain networks. Front Neuroinform 8, 12, doi:10.3389/fninf.2014.00012 (2014).

32 Watanabe, T., Masuda, N., Megumi, F., Kanai, R. \& Rees, G. Energy landscape and dynamics of brain activity during human bistable perception. Nature communications 5, 4765, doi:10.1038/ncomms5765 (2014).

33 Watanabe, T. \& Rees, G. Brain network dynamics in high-functioning individuals with autism. Nature communications 8, 16048, doi:10.1038/ncomms16048 (2017).

34 Esterman, M., Poole, V., Liu, G. \& DeGutis, J. Modulating Reward Induces Differential Neurocognitive Approaches to Sustained Attention. Cerebral cortex 27, 4022-4032, doi:10.1093/cercor/bhw214 (2017).

35 Esterman, M., Reagan, A., Liu, G., Turner, C. \& DeGutis, J. Reward reveals dissociable aspects of sustained attention. J Exp Psychol Gen 143, 2287-2295, doi:10.1037/xge0000019 (2014).

36 Kreutz-Delgado, K. et al. Dictionary learning algorithms for sparse representation. Neural computation 15, 349-396, doi:10.1162/089976603762552951 (2003).

37 Mensch, A., Varoquaux, G. \& Thirion, B. Compressed online dictionary learning for fast resting-state fMRI decomposition in 2016 IEEE 13th International Symposium on Biomedical Imaging (ISBI). 1282-1285 (IEEE).

38 Varoquaux, G., Gramfort, A., Pedregosa, F., Michel, V. \& Thirion, B. Multi-subject dictionary learning to segment an atlas of brain spontaneous activity. Information processing in medical imaging : proceedings of the ... conference $\mathbf{2 2}$, 562-573 (2011).

39 Watanabe, T. et al. A pairwise maximum entropy model accurately describes resting-state human brain networks. Nature communications 4, 1370, doi:10.1038/ncomms2388 (2013). 
40 Schaefer, A. et al. Local-Global Parcellation of the Human Cerebral Cortex from Intrinsic Functional Connectivity MRI. Cerebral cortex 28, 3095-3114, doi:10.1093/cercor/bhx179 (2018).

41 Power, J. D. et al. Functional network organization of the human brain. Neuron 72, 665-678, doi:10.1016/j.neuron.2011.09.006 (2011).

42 Christoff, K., Gordon, A. M., Smallwood, J., Smith, R. \& Schooler, J. W. Experience sampling during fMRI reveals default network and executive system contributions to mind wandering. Proceedings of the National Academy of Sciences of the United States of America 106, 8719-8724, doi:10.1073/pnas.0900234106 (2009).

43 Mason, M. F. et al. Wandering minds: the default network and stimulus-independent thought. Science 315, 393-395, doi:10.1126/science.1131295 (2007).

44 Vatansever, D., Menon, D. K. \& Stamatakis, E. A. Default mode contributions to automated information processing. Proceedings of the National Academy of Sciences of the United States of America 114, 12821-12826, doi:10.1073/pnas.1710521114 (2017).

45 Esterman, M. et al. Network-targeted cerebellar transcranial magnetic stimulation improves attentional control. NeuroImage 156, 190-198, doi:10.1016/j.neuroimage.2017.05.011 (2017).

46 deBettencourt, M. T., Cohen, J. D., Lee, R. F., Norman, K. A. \& Turk-Browne, N. B. Closed-loop training of attention with real-time brain imaging. Nature neuroscience 18, 470-475, doi:10.1038/nn.3940 (2015).

47 deBettencourt, M. T., Keene, P. A., Awh, E. \& Vogel, E. K. Real-time triggering reveals concurrent lapses of attention and working memory. Nat Hum Behav 3, 808-816, doi:10.1038/s41562-019-0606-6 (2019).

48 Taghia, J. et al. Uncovering hidden brain state dynamics that regulate performance and decision-making during cognition. Nature communications $\mathbf{9}, 2505$, doi:10.1038/s41467-018-04723-6 (2018).

49 Allan Cheyne, J., Solman, G. J., Carriere, J. S. \& Smilek, D. Anatomy of an error: a bidirectional state model of task engagement/disengagement and attention-related errors. Cognition 111, 98-113, doi:10.1016/j.cognition.2008.12.009 (2009).

50 Di Martino, A. et al. Decomposing intra-subject variability in children with attention-deficit/hyperactivity disorder. Biol Psychiatry 64, 607-614, doi:10.1016/j.biopsych.2008.03.008 (2008).

51 Esteban, O. et al. fMRIPrep: a robust preprocessing pipeline for functional MRI. Nature Methods 16, 111-116, doi:10.1038/s41592-018-0235-4 (2019).

52 Abraham, A. et al. Machine learning for neuroimaging with scikit-learn. Front Neuroinform 8, 14, doi:10.3389/fninf.2014.00014 (2014).

53 Ciric, R. et al. Benchmarking of participant-level confound regression 
1 strategies for the control of motion artifact in studies of functional connectivity. Neuroimage 154, 174-187, doi:10.1016/j.neuroimage.2017.03.020 (2017). correction method (CompCor) for BOLD and perfusion based fMRI. Neuroimage 37, 90-101, doi:10.1016/j.neuroimage.2007.04.042 (2007). Review 106, 620-630, doi:10.1103/PhysRev.106.620 (1957). Computing in Python. arXiv preprint arXiv:1907.10121 (2019). 